\title{
Minimum Electrical and Thermal Conductivity of Graphene: A Quasiclassical Approach
}

\author{
Maxim Trushin and John Schliemann \\ Institute for Theoretical Physics, University of Regensburg, D-93040 Regensburg, Germany
}

(Dated: October 25, 2018)

\begin{abstract}
We investigate the minimum conductivity of graphene within a quasiclassical approach taking into account electron-hole coherence effects which stem from the chiral nature of low energy excitations. Relying on an analytical solution of the kinetic equation in the electron-hole coherent and incoherent cases we study both the electrical and thermal conductivity whose relation fullfills Wiedemann-Franz law. We found that the most of the previous findings based on the Boltzmann equation are restricted to only high mobility samples where electron-hole coherence effects are not sufficient.
\end{abstract}

Introduction. Single graphite layers (graphene) have been found in the free state only recently [1], and their transport properties have immediately attracted much attention from both experimental $[2,3,44,5,6,7,8]$ and theoretical [9, 10, 11, 12, 13, 14, 15, 16] investigators. The reason of such an explosive interest is a number of very unusual transport properties including (i) a nonvanishing electrical conductivity even at zero carrier concentration (minimum conductivity phenomena), and (ii) independence of this minimum conductivity of temperature. Besides these unconventional transport properties many related phenomena have been studied in graphene such as weak localisation [15], the Klein paradox 16] etc. The remarkable electronic properties of graphene are usually attributed to the particular spectrum of excitations [17] which consists of two conical bands and is described by a two-dimensional analog of the relativistic Dirac equation. For a review concerning the history, fabrication, fundamental properties, and future applications of graphene we refer to the recent article [18].

To investigate the transport properties (i) and (ii) of graphene several different approaches have been applied including the Kubo formalism [9, 10, 11], direct calculations of the transmission probability for ballistic samples [11, 12], and Boltzmann equation [9, 13]. The latter approach looks at the first sight inapplicable for investigation of the minimum conductivity since the quasiclassical description is expected to fail at low Fermi energies $E_{F}$ as soon as $E_{F} \tau\left(E_{F}\right)$ becomes comparable with $\hbar$. The carrier momentum relaxation time $\tau$, on the other hand, diverges at $E_{F}=0[9]$ for short-range scatterers studied here as well. Thus, the product $E_{F} \tau\left(E_{F}\right)$ depends at $E_{F} \rightarrow 0$ on the scattering parameters rather than the carrier concentration and can acquire values much lager than $\hbar$ even at zero doping. In this Letter we solve the kinetic equation for Dirac fermions including off-diagonal elements of the distribution function in the helicity basis which are strongly connected with the electron-hole coherence or Zitterbewegung effects studied recently in graphene by Auslender and Katsnelson [19]. We find that the off-diagonal elements essentially contribute to the minimum conductivity in low mobil- ity samples, and, thus, the conclusions obtained in 13 are restricted to only quite perfect graphene sheets. To discuss our findings we adduce both the electron-hole coherent and conventional solutions of the kinetic equation. As an application the electrical and thermal conductivity is calculated.

Preliminaries. The carriers in the $\pi$-system of graphene near half filling can be described by the Dirac Hamiltonian

$$
H=\hbar v_{0}\left(\sigma_{x} k_{x}+\sigma_{y} k_{y}\right)
$$

where $v_{0} \approx 10^{6} \mathrm{~ms}^{-1}$ is the effective "speed of light", $\sigma_{x, y}$ are the Pauli matrices, and $\mathbf{k}$ is the two-component particle momentum. The eigenstates of (1) have the form

$$
\Psi_{\mathbf{k} \pm}(x, y)=\frac{1}{\sqrt{2}} \mathrm{e}^{i k_{x} x+i k_{y} y}\left(\begin{array}{c}
1 \\
\pm \mathrm{e}^{i \theta}
\end{array}\right)
$$

where $\tan \theta=k_{y} / k_{x}$, and the energy spectrum reads $E_{k \pm}= \pm \hbar v_{0} k$. The velocity matrix in the basis (2) is

$$
\frac{\mathbf{v}}{v_{0}}=\mathbf{e}_{x}\left(\begin{array}{cc}
\cos \theta & -i \sin \theta \\
i \sin \theta & -\cos \theta
\end{array}\right)+\mathbf{e}_{y}\left(\begin{array}{cc}
\sin \theta & i \cos \theta \\
-i \cos \theta & -\sin \theta
\end{array}\right) \text {. }
$$

Let us first consider the Boltzmann equation for charge carriers in the presence of impurity scatterers described by the effective potential

$$
V(\mathbf{r})=\frac{q e Z}{r} \mathrm{e}^{-r / R}
$$

where $e Z, q$ are the impurity atom and carrier electrostatic charge respectively, and $R$ is the screening radius. The potential (4) differs from its conventional short-range $\delta$-function approximation [9] by the additional fitting parameter $R$. As we shall see below, the minimum conductivity value is governed by both the impurity concentration and screening radius whereas the carrier mobility turns out to be $R$-independent. It seems to be necessary to introduce such a parameter in order to explain the experimental picture [8] where two samples made out of the same graphene flake (i. e. having equal mobility) demonstrate essentially different minimum conductivity values. 
This difference is attributed to the screening parameter $R$ in our model.

Electron-hole incoherent solution. In linear order in the homogeneous electric field $\mathbf{E}$ the Boltzmann equation reads

$$
\left(\frac{d f_{\kappa}}{d t}\right)^{\text {coll }}=-q \mathbf{E v}_{\kappa}\left[-\frac{\partial f^{0}\left(E_{k \kappa}\right)}{\partial E_{k \kappa}}\right]
$$

where we have divided the distribution function $f_{\kappa}(\mathbf{k})=$ $f^{0}\left(E_{k \kappa}\right)+f_{\kappa}^{1}(\mathbf{k})$ into an equilibrium contribution $f^{0}\left(E_{k \kappa}\right)$ and a nonequilibrium part $f_{\kappa}^{1}(\mathbf{k})$, and $\mathbf{v}_{\kappa}$ (with $\kappa \in\{ \pm\}$ ) are the diagonal elements of the velocity operator in the helicity basis, cf. Eq. (3). Assuming elastic scattering fulfilling the microreversibility condition, the collision term can be written as

$$
\left(\frac{d f_{\kappa}}{d t}\right)^{\text {coll }}=\sum_{\kappa^{\prime}} \int \frac{d^{2} k^{\prime}}{\pi^{2}}\left\{w\left(\mathbf{k} \kappa, \mathbf{k}^{\prime} \kappa^{\prime}\right)\left[f_{\kappa^{\prime}}\left(\mathbf{k}^{\prime}\right)-f_{\kappa}(\mathbf{k})\right]\right\},
$$

where the scattering probability $w\left(\mathbf{k} \kappa, \mathbf{k}^{\prime} \kappa^{\prime}\right)$ can be easily found from Fermi's golden rule,

$$
\begin{aligned}
w\left(\mathbf{k} \kappa, \mathbf{k}^{\prime} \kappa^{\prime}\right)= & \frac{4 \pi R^{2} V_{0}^{2}}{\hbar} \delta\left(E_{k \kappa}-E_{k^{\prime} \kappa^{\prime}}\right) \times \\
& \frac{1+\kappa \kappa^{\prime} \cos \left(\theta^{\prime}-\theta\right)}{1+R^{2}\left[k^{2}+k^{\prime 2}-2 k k^{\prime} \cos \left(\theta^{\prime}-\theta\right)\right]} .
\end{aligned}
$$

Here $V_{0}=\pi q e Z \sqrt{N}$ is an effective scattering potential with $N$ being the impurity concentration, and the fourfold (valley and spin) degeneracy is taken into account by the factor 4 in the integrand. The exact analytical solution of Eq. (5) can be given in terms of the nonequilibrium part of the distribution function $f_{\kappa}^{1}=$ $q \mathbf{E v}_{\kappa} \tau(k)\left[-\partial f^{0}\left(E_{k \kappa}\right) / \partial E_{k \kappa}\right]$ with $\tau(k)$ given by

$$
\begin{aligned}
\tau(k) & =\frac{1}{k} \frac{\hbar^{2} v_{0}}{4 R^{2} V_{0}^{2}} \frac{2 R^{4} k^{4}}{1+2 R^{2} k^{2}-\sqrt{1+4 R^{2} k^{2}}} \\
& \approx \frac{1}{k} \frac{\hbar^{2} v_{0}}{4 R^{2} V_{0}^{2}}, \quad R k \ll 1 .
\end{aligned}
$$

Electron-hole coherent solution. So far we have neglected the off-diagonal elements of the distribution function, an valid approximation if the decoherence of the single-carrier state is a fast process compared to its relaxation described by the above equations. In general, a particle described by the Hamiltonian (11) can not only be in one of the states $\Psi_{\mathbf{k}+}$ or $\Psi_{\mathbf{k}-}$ but in an arbitrary superposition of them. Therefore, generalising the above considerations, the distribution function is $2 \times 2$ nondiagonal matrix $\hat{f}(\mathbf{k})$, and the kinetic equation contains the commutator $\frac{i}{\hbar}[H, \hat{f}(\mathbf{k})]$, which drops out if only the diagonal elements of $\hat{f}(\mathbf{k})$ (with respect to the helicity basis) are retained. In the linear response regime the kinetic equation explicitly reads

$$
\begin{aligned}
& \left(\frac{d \hat{f}}{d t}\right)^{\text {coll }}=\frac{i}{\hbar}\left(\begin{array}{cc}
0 & f_{12}\left(E_{k+}-E_{-}\right) \\
f_{12}\left(E_{k+}-E_{-}\right) & 0
\end{array}\right) \\
& +q \mathbf{E}\left(\begin{array}{cc}
-\mathbf{v}_{11}\left[-\frac{\partial f^{0}\left(E_{k+}\right)}{\partial E_{k+}}\right] & \frac{\mathbf{v}_{12}}{2 E_{k+}}\left(f_{E_{k-}}^{0}-f_{E_{k+}}^{0}\right) \\
\frac{\mathbf{v}_{21}}{2 E_{k-}}\left(f_{E_{k+}}^{0}-f_{E_{k-}}^{0}\right) & -\mathbf{v}_{22}\left[-\frac{\partial f^{0}\left(E_{k-}\right)}{\partial E_{k-}}\right]
\end{array}\right)
\end{aligned}
$$

with the collision term given by the generalised expression [20]

$$
\begin{aligned}
& \left(\frac{d \hat{f}}{d t}\right)_{\kappa \kappa_{1}}^{\mathrm{coll}}=\int \frac{d^{2} k^{\prime}}{\pi^{2}} \sum_{\kappa^{\prime}, \kappa_{1}^{\prime}}\left\{\left[\delta\left(E_{k^{\prime} \kappa^{\prime}}-E_{k \kappa}\right)\right.\right. \\
& \left.+\delta\left(E_{k^{\prime} \kappa_{1}^{\prime}}-E_{k \kappa}\right)\right] K_{\kappa^{\prime} \kappa_{1}^{\prime}}^{\kappa \kappa_{1}} f_{\kappa^{\prime} \kappa_{1}^{\prime}}\left(k^{\prime}\right)-\delta\left(E_{k \kappa^{\prime}}-E_{k^{\prime} \kappa_{1}^{\prime}}\right) \\
& \left.\times\left[K_{\kappa_{1}^{\prime} \kappa_{1}^{\prime}}^{\kappa \kappa^{\prime}} f_{\kappa^{\prime} \kappa_{1}}(k)+K_{\kappa_{1}^{\prime} \kappa_{1}^{\prime}}^{\kappa^{\prime} \kappa_{1}} f_{\kappa_{1}^{\prime} \kappa_{1}^{\prime}}(k)\right]\right\},
\end{aligned}
$$

and $K_{\kappa^{\prime} \kappa_{1}^{\prime}}^{\kappa \kappa_{1}}$ being

$$
\begin{aligned}
& K_{\kappa^{\prime} \kappa_{1}^{\prime}}^{\kappa \kappa_{1}}=\left(\pi R^{2} V_{0}^{2} / \hbar\right) \\
& \times \frac{1+\kappa \kappa^{\prime} \kappa_{1} \kappa_{1}^{\prime}+\kappa \kappa^{\prime} \mathrm{e}^{i\left(\theta^{\prime}-\theta\right)}+\kappa_{1} \kappa_{1}^{\prime} \mathrm{e}^{-i\left(\theta^{\prime}-\theta\right)}}{1+R^{2}\left[k^{2}+k^{2}-2 k k^{\prime} \cos \left(\theta^{\prime}-\theta\right)\right]} .
\end{aligned}
$$

Note, that the collision term includes off-diagonal elements of $\hat{f}$ and in that way significantly enhances the complexity of the kinetic equation. However, by somewhat more tedious calculations one can again construct an analytical solution to Eq. (10) with nonequilibrium terms given by

$$
\begin{aligned}
& f_{11}^{1}=q \mathbf{E v}_{11} \tau(k)\left\{\left(1+\frac{1}{2 \alpha}\right)\left[-\frac{\partial f^{0}\left(E_{k+}\right)}{\partial E_{k+}}\right]\right. \\
& \left.+\frac{1}{2 \alpha}\left[-\frac{\partial f^{0}\left(E_{k-}\right)}{\partial E_{k-}}\right]+\frac{1}{2 \alpha E_{k+}}\left(f_{E_{k+}}^{0}-f_{E_{k-}}^{0}\right)\right\}(13) \\
& f_{12}^{1}=\frac{q \mathbf{E v}_{12} \tau(k)\left(\frac{1}{2}+\frac{1}{2 \alpha}\right)}{1+2 i E_{k+} \tau(k) / \hbar}\left\{\frac{1}{E_{k+}}\left(f_{E_{k+}}^{0}-f_{E_{k-}}^{0}\right)\right. \\
& \left.+\left[-\frac{\partial f^{0}\left(E_{k+}\right)}{\partial E_{k+}}-\frac{\partial f^{0}\left(E_{k-}\right)}{\partial E_{k-}}\right]\right\},
\end{aligned}
$$

and $f_{22}^{1}, f_{21}^{1}$ can be obtained from Eqs. (13) 14) just exchanging the indices belong to $E_{k}$ and $\mathbf{v}$ accordingly. Here we have introduced the novel electron-hole incoherence parameter $\alpha=4 E_{k+}^{2} \tau^{2}(k) / \hbar^{2}$. In order to simplify the solution we use $\tau(k)$ given by Eq. (9), thus, $\alpha=\hbar^{4} v_{0}^{4} / 4 R^{4} V_{0}^{4}$ is independent on $k$. In the limit case of weak scattering $(\alpha \gg 1)$ the diagonal elements $f_{11}^{1}$ and $f_{22}^{1}$ are the same as in the electron-hole incoherent case and given by $f_{\kappa}^{1}, \kappa \in\{ \pm\}$, whereas the off-diagonal elements $f_{12}^{1}$ and $f_{21}^{1}$ are real and, as we shall see from Eq. (15), do not contribute to the current.

Electrical conductivity. The electrical current reads

$$
\mathbf{j}=4 q \int \frac{d^{2} k}{(2 \pi)^{2}}\left(\mathbf{v}_{\mathbf{1 1}} f_{11}+\mathbf{v}_{\mathbf{2 2}} f_{22}+2 \Im \mathbf{v}_{\mathbf{1 2}} \Im f_{12}\right),
$$


where the factor 4 is due to the fourfold degeneracy.

Let us first concentrate on the electron-hole incoherent case when $\alpha \gg 1$. Utilising Eq. (9) we find from Eq. (15) for the conductivity

$$
\sigma \equiv \sigma_{0}=\frac{q^{2} \hbar v_{0}^{2}}{4 \pi R^{2} V_{0}^{2}} \equiv \frac{q^{2} \sqrt{\alpha}}{h},
$$

which, in particular, does neither depend on temperature nor on the carrier concentration. Thus, the most striking features of the experimental findings are reproduced: (i) the conductivity is not zero even at zero carrier concentration, (ii) this minimum conductivity does not depend on temperature. Note, that the above result is not universal, i.e. the minimum conductivity at zero Fermi energy can change from sample to sample in accordance with recent experimental reports [7, 8, 21]. Moreover $\sqrt{\alpha}>4$ (i.e. $1 / \alpha<0.0625)$ for the vast majority of samples 7, 8].

Let us take into account higher-order terms in $\tau(R k)$. Then instead of Eq. (16) we have at zero temperature

$$
\begin{aligned}
\sigma & =\frac{q^{2}}{\pi \hbar^{2}} \tau\left(k_{F}\right) E_{F}, \\
& \approx \sigma_{0}+q^{2} \frac{\hbar v_{0}^{2}}{2 V_{0}^{2}} n, \quad 2 \pi R^{2} n \ll 1
\end{aligned}
$$

where the carrier (electron) concentration is given by $n=k_{F}^{2} / \pi$ with $k_{F}=E_{F} / \hbar v_{0}$ being the Fermi wave vector. Thus, the low-temperature conductivity at low doping increases linearly with carrier concentration, in accordance with the experiments. Deviations from linear dependency can be described taking into account $k$ dependence of the relaxation time given by Eq. (8). We emphasise, that in contrast with [8] we deal with two fitting parameters $R$ and $V_{0}$ which can be deduced using Eq. (18) and the experimental data [2, 7, 8]. Indeed, from Eq. (18) we can define the electron mobility as $\mu=q \hbar v_{0}^{2} /\left(2 V_{0}^{2}\right)$ which does not depend on the screening radius $R$. The effective scattering potential then reads $V_{0}=v_{0} \sqrt{q \hbar /(2 \mu)}$, and for the most common samples with $\mu$ ranged from $\sim 10^{3}$ to $2 \cdot 10^{4} \mathrm{~cm}^{2} /(\mathrm{Vs})$ we have $V_{0}$ covering the range from $0.1 \mathrm{meV}$ to $0.06 \mathrm{eV}$. The screening radius can be estimated from Eq. (16) assuming that $\sigma_{0}$ is of the order of $4 q^{2} / h$. Then we have $R$ of the order of $\hbar v_{0} / V_{0}$ covering the range from $10^{-3} \mathrm{~cm}$ (high mobility samples) to $10^{-6} \mathrm{~cm}$ (low mobility samples). Now one can see that the linear approximation (18) in terms of $2 \pi R^{2} n$ at $n \sim 10^{12} \mathrm{~cm}^{-2}$ holds only in relatively low mobility samples, in accordance with the experimental report [7, 8]. The difference between $\sigma(n)$ and its linear approximation can be seen in Fig. 1.

To consider the low mobility samples properly, we should take into account the terms proportional to $1 / \alpha$ in the solution (13 14). Note, above all, that the quasiclassical approach is doubtful at $\alpha \leqslant 1$ since $E_{F} \tau\left(k_{F}\right) \leqslant \hbar$, and the carrier mean free path becomes comparable with

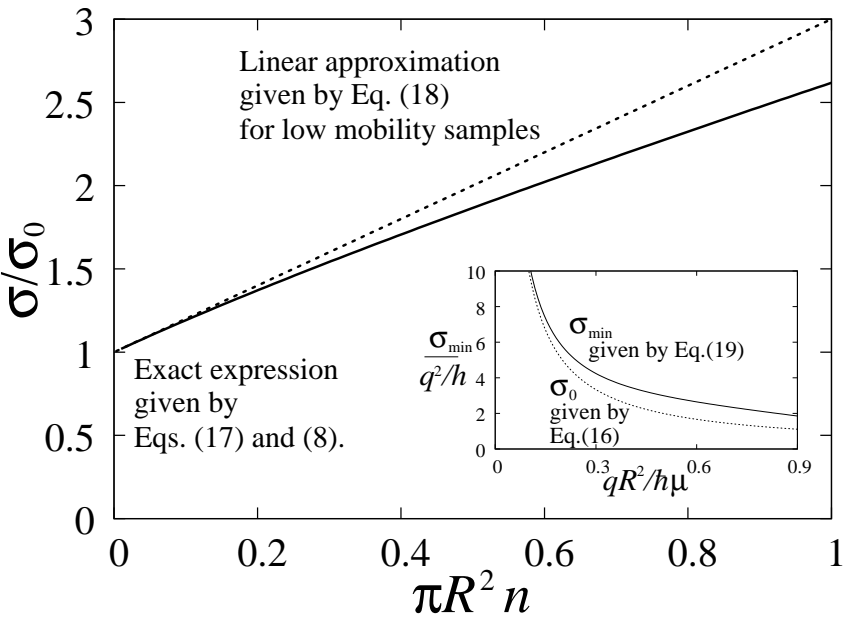

FIG. 1: Conductivity dependence on the carrier concentration, cf. Ref. [8]. Inset: the minimum conductivity vs. inverse mobility, cf. Ref. [7]. In order to preserve the validity of the quasiclassical description the parameters are chosen so that $\alpha>1$. The electron-hole coherence correction is nevertheless clearly seen.

its de Broglie wavelength. Nevertheless, an asymptotic dependence of the solution close to $\alpha \sim 1$ can give us a clue to what happens in this regime. The direct integration of Eq. (15) leads to the logarithmic divergence due to the terms proportional to $f_{E_{k+}}^{0}-f_{E_{k-}}^{0}$ in Eqs. (13 14). This problem has been solved in Ref. [19] introducing some ultraviolet cut off energy $E_{c}$ which allows to get a finite value for the conductivity but still has an unclear physical meaning itself. In our opinion the divergence of the integral in Eq. (15) is a clear manifestation of obvious limitations inherent in the quasiclassical approach at $E_{F} \tau\left(k_{F}\right) \sim \hbar$. Thus, we expect the ultraviolet cut off $E_{c}$ to have a quantum mechanical origin. At $E_{F}=0$ and zero temperature the quasiparticle energy will fluctuate around $E=0$ with a variance $\Delta E$ related to the relaxation time via the uncertainty relation $\Delta E \tau \sim \hbar$. Obviously, $E_{c}$ is the maximum energy uncertainty consistent with two subsequent scattering events ("measurements") separated by a time interval $\tau\left(k_{F}\right)$, i. e. $E_{c} \sim \hbar \tau^{-1}\left(k_{F}\right)$. Then Eq. (15) can be integrated easily, and the minimum conductivity for low mobility samples reads

$$
\sigma_{\min }=\sigma_{0}\left[1+\frac{2}{\alpha}\left(1-\frac{1}{2} \ln \left|\frac{4}{\alpha}\right|\right)\right] .
$$

The difference between $\sigma_{\min }$ and $\sigma_{0}$ given by Eq. (16) is shown in Fig. 1 (inset). We emphasise that Eq. (19) could not be well mathematically grounded in the framework of our quasiclassical model because of quantum effects which obviously contribute to the conductivity minimum at $\alpha \sim 1$. What is certainly true is that the additional term in the conductivity minimum stemming from the electron-hole coherence increases in low mobility samples and partly compensates the diminution of the lead- 
ing term given by Eq. (16). This mechanism might be responsible for the non-monotonic dependence of the conductivity minimum on the impurity concentration (inverse mobility) observed recently [7].

We have also studied the conductivity choosing potential profiles different from one given by Eq. (44). To give an example for hard-wall potential when $V(\mathbf{r})=U_{0}$ at $r \leq r_{0}$, and $V(\mathbf{r})=0$ at $r>r_{0}$, we obtain for the conductivity the same formulas as before besides the substitutions $V_{0} \rightarrow \pi r_{0} \sqrt{N} U_{0}$ and $R \rightarrow r_{0} / 2$. The mobility becomes $r_{0}$-dependent in this case that makes it difficult to fit our model to the experimental data [8]. Most interesting is, however, $\delta$-function shaped scattering potential which can be used as a model for neutral impurities or lattice imperfections. In this case the Boltzmann equation (5) can be solved easily, and the conductivity does not depend on the carrier concentration at all, what, in turn, contradicts the measurements. Thus, our shortrange potential choice given by Eq. (4) fits best to nowadays experimental data.

Thermal conductivity. Following our method it is possible to show that the thermal conductivity of graphene also has a minimal value which does not depend on the concentration, but depends on the temperature according to the Wiedemann-Franz law as was pointed out in Refs. [22, 23]. Indeed, in presence of the temperature gradient $\nabla T$ we have at $\alpha \gg 1$ in the linear response regime $f_{\kappa}^{1}=(\nabla T / T) \mathbf{v}_{\kappa} \tau(k)\left(E_{k \kappa}-E_{F}\right)\left[-\partial f^{0}\left(E_{k \kappa}\right) / \partial E_{k \kappa}\right]$. The further calculations of the thermal flow are very similar to that for the electrical current. In particular, the minimum thermal conductivity takes the form

$$
\sigma^{\text {th }}\left(E_{F}=0\right) \equiv \sigma_{0}^{\text {th }}=\frac{\pi \hbar v_{0}^{2}}{12 R^{2} V_{0}^{2}} T .
$$

The influence of electron-hole coherence on $\sigma_{0}^{\text {th }}$ can be described by Eq. (19) in full analogy with the electrical conductivity.

Conclusions. We have solved the quasiclassical kinetic equation for carriers in a single graphene sheet including the off-diagonal elements of the distribution function in the helicity basis. The analytical solution allows us to investigate the influence of the electron-hole coherence on the minimum conductivity phenomena as well as to discover the limitations of previous studies based on Boltzmann equation. We have introduced a special parameter $\alpha$ distinguishing the electron-hole coherent and incoherent regimes. It is noteworthy that $\alpha$ can be deduced directly from the minimum conductivity measurements since it is incorporated into $\sigma_{0}$ in a simple way given by Eqs. (16), (19). Moreover, our approach successfully describes the linear dependence of the conductivity above its minimum which is usual for low mobility samples. Finally, we predicted the existence of the thermal conductivity minimum which was not observed so far.

Acknowledgements. We thank Daniel HuertasHernando, Inanc Adagideli, Shaffique Adam and Kostya
Novoselov for fruitful and stimulating discussions. This work was financially supported by SFB 689 .

[1] K. S. Novoselov, A. K. Geim, S. V. Morozov, D. Jiang, Y. Zhang, S. V. Dubonos, I. V. Grigorieva, and A. A. Firsov, Science 306, 666 (2004).

[2] K. S. Novoselov, A. K. Geim, S. V. Morozov, D. Jiang, M. I. Katsnelson, I. V. Grigorieva, S. V. Dubonos, and A. A. Firsov, Nature 438, 197 (2005).

[3] Y. Zhang, Y.-W. Tan, H. L. Stormer, and P. Kim, Nature 438, 201 (2005).

[4] S. V. Morozov, K. S. Novoselov, M. I. Katsnelson, F. Schedin, L. A. Ponomarenko, D. Jiang, and A. K. Geim, Phys. Rev. Lett. 97, 16801 (2006).

[5] F. Miao, S. Wijeratne, Y. Zhang, U. C. Coskun, W. Bao, and C. N. Lau, Science 317, 1530 (2007).

[6] S. Cho and M. S. Fuhrer arXiv:0705.3239.

[7] J. H. Chen, C. Jang, M. S. Fuhrer, E. D. Williams, M. Ishigami arXiv:0708.2408.

[8] Y.-W. Tan, Y. Zhang, K. Bolotin, Y. Zhao, S. Adam, E.H. Hwang, S. Das Sarma, H. L. Stormer, P. Kim arXiv:0707.1807.

[9] K. Nomura and A. H. MacDonald, Phys. Rev. Lett. 98, 76602 (2007).

[10] K. Ziegler, Phys. Rev. Lett. 97, 266802 (2006), P. M. Ostrovsky, I. V. Gornyi, and A. D. Mirlin, Phys. Rev. B 74, 235443 (2006), N. M. R. Peres, F. Guinea, and A. H. C. Neto, ibid 73, 125411 (2006), J. Cserti, ibid 75, 33405 (2007), V. P. Gusynin and S. G. Sharapov, ibid 71, 125124 (2005), V. P. Gusynin and S. G. Sharapov, ibid 73, 245411 (2006).

[11] M. I. Katsnelson, Euro Phys. J. B 51, 157 (2006).

[12] J. Tworzydlo, B. Trauzettel, M. Titov, A. Rycerz, and C. W. J. Beenakker, Phys. Rev. Lett. 96, 246802 (2006), A. R. Akhmerov and C. W. J. Beenakker, Phys. Rev. B 75, 45426 (2007), J. P. Robinson and H. Schomerus, ibid 76, 115430 (2007), H. Schomerus, ibid 76, 45433 (2007).

[13] K. Nomura and A. H. MacDonald, Phys. Rev. Lett. 96, 256602 (2006), E. H. Hwang, S. Adam, and S. D. Sarma, ibid 98, 186806 (2007), T. Ando, J. Phys. Soc. Jpn 75, 74716 (2006), T. Stauber, N. M. R. Peres, F. Guinea arXiv:0707.3004, S. Adam, E. H. Hwang, V. M. Galitski, S. Das Sarma arXiv:0705.1540.

[14] A. Altland, Phys. Rev. Lett 97, 236802 (2006).

[15] I. L. Aleiner and K. B. Efetov, Phys. Rev. Lett. 97, 236801 (2006), D. V. Khveshchenko, ibid 97, 36802 (2006), E. McCann, K. Kechedzhi, V. I. Fal'ko, H. Suzuura, T. Ando, and B. L. Altshuler, ibid 97, 146805 (2006).

[16] M. I. Katsnelson, K. S. Novoselov, and A. K. Geim, Nat. Phys. 2, 620 (2006).

[17] P. R. Wallace, Phys. Rev. 71, 622 (1947).

[18] A. K. Geim and K. S. Novoselov, Nat. Mat. 6, 183 (2007).

[19] M. Auslender, M. I. Katsnelson arXiv:0707.2804.

[20] M. I. D'yakonov and A. V. Khaetskii, JETP 59, 1072 (1984).

[21] K. Novoselov, priv. comm.

[22] V. P. Gusynin and S. G. Sharapov, Phys. Rev. B 71, 125124 (2005).

[23] B. Dora and P. Thalmeier arXiv:0701714. 